\title{
Vagus Nerve Stimulation in Experimental Heart Failure
}

\author{
Hani N. Sabbah, Ph.D., Itamar Ilsar, DVM, Asaph Zaretsky, DVM ${ }^{1}$, Sharad Rastogi, M.D., \\ Mengjun Wang, M.D., and Ramesh C. Gupta, Ph.D. \\ Department of Medicine, Division of Cardiovascular Medicine, Henry Ford Heart \& Vascular \\ Institute, Henry Ford Hospital, Detroit, Michigan \\ ${ }^{1}$ Technion IIT, Haifa, Israel
}

\begin{abstract}
Chronic heart failure (HF) is associated with autonomic dysregulation characterized by a sustained increase of sympathetic drive and by withdrawal of parasympathetic activity. Sympathetic overdrive and increased heart rate are predictors of poor long-term outcome in patients with HF. Considerable evidence exists that supports the use of pharmacologic agents that partially inhibit sympathetic activity as effective long-term therapy for patients with HF; the classic example is the wide use of selective and non-selective beta-adrenergic receptor blockers. In contrast, modulation of parasympathetic activation as potential therapy for HF has received only limited attention over the years given its complex cardiovascular effects. In this article, we review results of recent experimental animal studies that provide support for the possible use of electrical Vagus nerve stimulation (VNS) as a long-term therapy for the treatment of chronic HF. In addition to exploring the effects of chronic VNS on left ventricular (LV) function, the review will also address the effects of VNS on potential modifiers of the HF state that include cytokine production and nitric oxide elaboration. Finally, we will briefly review other nerve stimulation approaches also currently under investigation as potential therapeutic modalities for treating chronic HF.
\end{abstract}

\section{Keywords}

Ventricular function; Ventricular remodeling; Electrical nerve stimulation; Sympathetic activity; Parasympathetic activity; Animal models of disease; Cytokines; Nitric oxide

\section{Introduction}

Autonomic dysregulation is a characteristic feature of heart failure (HF) manifested by increased sympathetic activity and reduced parasympathetic activity. These abnormalities in autonomic regulation have long been recognized as mediators of increased mortality and morbidity in myocardial infarction and $\operatorname{HF}(1,2)$. Results from the Autonomic Tone and Reflexes after Myocardial Infarction study (ATRAMI) and the Cardiac Insufficiency Bisoprolol Study II (CIBIS II) showed that diminished vagal activity and increased heart rate are predictors of high mortality in $\operatorname{HF}(3,4)$. In addition to increasing mortality, the sustained increased sympathetic activity and reduced parasympathetic activity also contribute to progressive left ventricular (LV) dysfunction in HF and promote progressive LV remodeling leading to intractable HF. Numerous studies in experimental models of HF in which the long-term use of beta-blockers and other anti-adrenergic agents and more recently from use of specific and selective agents that reduce heart rate through inhibition of

Address for Correspondence: Hani N. Sabbah, PhD, Director, Cardiovascular Research, Henry Ford Hospital, 2799 West Grand Boulevard, Detroit, Michigan 48202, Office Phone: (313) 916-7360, Office Fax: (313) 916-3001, hsabbah1@hfhs.org. 
the cardiac pacemaker current I $f$ have shown that these agents can prevent or attenuate progressive LV remodeling (5-8).

While much of the emphasis in treating HF has over the past decade or two focused on modulation of sympathetic activity, considerable interest has emerged recently in modulating parasympathetic or vagal activity as a therapeutic target for treating chronic HF. It has long been recognized that alteration in cardiac vagal efferent activity through peripheral cardiac nerve stimulation can produce bradycardia (9), and modification in atrial $(10,11)$ ventricular contractile function $(12,13)$. In 1991, Vanoli and colleagues showed that electrical Vagus nerve stimulation (VNS) can prevent sudden cardiac death in conscious dogs with a healed myocardial infarction (14) and in 2004, Li et al showed that VNS markedly improved long-term survival after chronic HF in rats (15). Consistent with these findings, VNS has also been shown to markedly suppress arrhythmias in conscious rats with chronic HF secondary to myocardial infarction (16). The discussion that follows will focus primarily on the effects of chronic VNS on LV dysfunction and remodeling in canines with HF produced by multiple sequential intracoronary microembolizations (17) and canines with HF produced by high-rate ventricular pacing (18). In dogs with microembolization-induced HF, the CardioFit ${ }^{\mathrm{TM}}$ system (BioControl Medical Ltd., Yehud, Israel) was used to deliver VNS; whereas in the high-rate pacing studies, the Cyberonics system (Cyberonics Inc., Houston, TX), originally approved for drug resistant epilepsy, was used (18).

\section{The CardioFit System}

There are two types of efferent cardioinhibitory fibers in the cervical Vagus namely small myelinated $\mathrm{A} \delta$ (or B) fibers and non-myelinated $\mathrm{C}$ fibers (19). The minimal current required to activate $\mathrm{B}$ fibers with a cuff electrode and thereby, presumably increase parasympathetic tone, was shown in anesthetized pigs to be in the range of 1.5 to $2.0 \mathrm{~mA}$ range (20). While the 1.5 to $2.0 \mathrm{~mA}$ range is sufficient to start activating $\mathrm{B}$ fibers, the recruitment of a larger proportion of B type nerve fibers is necessary and hence a higher current is required to elicit the potentially beneficial effect of vagal stimulation. However, side effects limit the current to that level and seldom allow higher amplitudes as previously shown in epilepsy and depression patients with implanted VNS devices (21-24). Hence, in order to design an electrode to stimulate cardioinhibitory vagal B fibers, care should be given to minimize any uncontrolled effects on the central circuitry that could arise from propagation of neural activation toward the cranium caused by vagal stimulation of lower threshold fibers. The CardioFit ${ }^{\mathrm{TM}}$ Stimulation Lead (CSL) is a modified bi-polar cuff electrode designed primarily for VNS (but that can be applied broadly) (Fig. 1). The CSL activates cardioinhibitory B fibers, while maintaining a large degree of unidirectionality with respect to low threshold fibers recruited in the 1-2 mA range. This selectively unidirectional activation is a property not shared by the helical cuff electrode widely used in VNS.

The CardioFit VNS system used in the studies discussed in this review delivered electrical stimulation to the right cervical Vagus only when heart rate increased beyond a pre-set level thus operating on a negative-feedback loop. The CardioFit VNS system consisted of a model 5000 electrostimulator (Fig. 1). The stimulator or generator was fitted with a processing unit that adjusted the impulse rate and intensity to keep the heart rate within the desired range. Maximal stimulation current, pulse width and operation algorithm are controlled by the Physician Programmer via wireless communication. A standard pacemaker bi-polar ventricular electrode was used in all the discussed animal studies for sensing the intracardiac electrocardiogram. The CSL stimulation lead and the sensing lead were connected to the nerve stimulator via a $3.2 \mathrm{~mm}$ IS-1 like connector. A model 5300 BioControl physician programmer was used in conjunction with the nerve stimulator to non-invasively adjust the stimulation algorithm and settings for optimal outcome of therapy in each individual case. 
Adjustments were made while the programming wand was placed over the implanted nerve stimulator. A model 5420 BioControl safety magnet was available and was used to turn the nerve stimulator off, if needed.

\section{VNS Study Protocols in Dogs with Microembolization-Induced HF}

Two separate studies were conducted in which the CardioFit system was used in dogs with microembolization induced HF. In the first study, 13 dogs with a LV ejection fraction of approximately $35 \%$ were studied. Seven dogs were randomized to active monotherapy (CardioFit ON) and 6 to no therapy at all (Sham-operated control, CardioFit OFF). All dogs were followed for 3 months. In all dogs, a bi-polar nerve cuff electrode was surgically implanted on the right Vagus nerve at the mid cervical position. In the active group, a signal generator was implanted along with a right ventricular sensing lead and the Cardiofit device was turned on after an on/off test was performed. The feedback on-demand heart rate control was set to reduce basal heart rate by $10 \%$. A generator and a right ventricular sensing lead were not implanted in control dogs. Data were collected just prior to CardioFit activation and again at the end of 3 months of follow-up (25).

In the second study, a similar cohort of dogs $(\mathrm{n}=12)$ were randomized to 3 months therapy with a beta-blocker alone (metoprolol succinate, $100 \mathrm{mg}$ once daily, $\mathrm{n}=6$ ) or to metoprolol (100 mg once daily) combined with active VNS with CardioFit $(n=6)$. As in the first study, the CardioFit VNS system was operated in the feedback on-demand heart rate responsive mode. All dogs were treated with metoprolol for 2 weeks before randomization to VNS therapy, continued to receive metoprolol succinate once daily for the entire duration of the study (26).

\section{Effects of VNS with the CardioFit System on LV Function and Remodeling}

In dogs with microembolization-induced HF, long-term VNS monotherapy significantly improved LV ejection fraction assessed ventriculographically and significantly decreased LV end-systolic and end-diastolic volumes compared to controls (Fig. 2) (25). These functional benefits were associated with changes of minimum, average and maximum heart rate assessed over the course of 3 months from ambulatory ECG Holter monitoring. In VNS treated dogs, minimum, average and maximum heart rate decreased by 1, 10, and 28 beats/ min respectively compared to changes of heart rate in control dogs of 2,1 and 0.5 beats $/ \mathrm{min}$ respectively.

The improvement in LV systolic function following long-term VNS therapy was accompanied by improvement in indices of LV diastolic function. VNS significantly decreased LV end-diastolic pressure compared to control (Table 1). VNS also significantly increased deceleration time of rapid mitral inflow velocity, tended to increase the ratio of peak mitral inflow velocity during early $\mathrm{LV}$ filling to peak mitral inflow velocity during left atrial contraction (PE/PA) and significantly reduced LV end-diastolic circumferential wall stress, a determinant of myocardial oxygen consumption (Table 1). These parameters, when viewed in concert, suggest that VNS can reduce preload, improve LV relaxation and potentially improve LV function without increasing myocardial oxygen consumption, all of which are desirable features in the treatment of chronic HF. The improvement in LV systolic and diastolic function and the reduction of LV volumes seen in VNS-treated dogs compared to untreated controls are consistent with the finding that plasma levels of $n$-terminal probrain natriuretic peptide (nt-pro BNP) decrease following VNS therapy compared to levels measured prior to initiating therapy and levels measured in untreated controls (Table 2).

In HF dogs receiving background therapy with beta-blockade (metoprolol succinate, $100 \mathrm{mg}$ once daily), the addition of VNS to beta-blockade significantly increased LV ejection 
fraction and significantly decreased LV end-systolic volume compared to dogs treated with beta-blockade alone (Fig. 3) (26). There were no significant differences in minimum and average heart rate between dogs treated with beta-blocker alone and those that received combination therapy with VNS. However, maximum heart rate was significantly lower in dogs treated with the combination therapy $(114 \pm 12$ beats/min) compared to those treated with a beta-blocker alone $(149 \pm 8$ beats/min, $\mathrm{p}<0.05)$. These data suggest that preventing heart rate escape at the high end (reducing periods of increased cardiac workload and increased myocardial oxygen consumption) in dogs treated with VNS in combination with beta-blockade may elicit added benefits in the form of improved LV systolic function.

\section{Effects of VNS with the CardioFit on Cellular Measures of LV Remodeling}

In dogs with coronary microembolization-induced HF, long-term VNS therapy elicited important changes in cellular and structural markers of LV remodeling. Histomorphometric assessment was performed at the end of 3 months of follow-up and included LV volume fraction of replacement fibrosis (VFRF), volume fraction of reactive interstitial fibrosis (VFIF), myocyte cross-sectional area (MCSA), a measure of cardiomyocyte hypertrophy, capillary density (CD), and oxygen diffusion distance (ODD) defined as half the distance between two adjoining capillaries $(6,27)$. Compared to normal dogs, control HF dogs showed a significant increase in VFRF, VFIF, ODD and MCSA and a significant decrease of CD (Table 3). Compared to control, treatment with VNS was associated with decreased VFRF, VFIF, ODD and MCSA and a significant increase of CD (Table 3). These observations suggest that VNS helps preserve myocardial structural integrity through direct or indirect action on the failing myocardium.

\section{Effects of VNS on Pro-Inflammatory Cytokines in Dogs with Heart Failure}

Increased elaboration of pro-inflammatory cytokines occurs in $\mathrm{HF}$ and is associated with increased morbidity and mortality. Electrical VNS has been shown to decrease the release of various cytokines including tumor necrosis factor-alpha (TNF- $\alpha$ ) and interlukin-6 (IL-6) $(28,29)$. In dogs with microembolization-induced HF, LV tissue levels of TNF- $\alpha$ and IL-6 are significantly elevated compared to LV tissue from normal dogs (Fig. 4). Long-term monotherapy with VNS has been shown normalized protein expression of both TNF- $\alpha$ and IL-6 in LV myocardium (Fig. 4). Plasma levels of TNF-a and IL-6 are also elevated in HF dogs compared to normal dogs and are attenuated following long-term monotherapy with VNS (30).

\section{Effects of VNS on Nitric Oxide Synthase Regulation in Dogs with Heart Failure}

Nitric oxide (NO) is formed from the guanidine group of L-arginine in an NADPHdependent reaction catalyzed by a family of nitric oxide synthases (NOS). Three isoforms of NOS have been identified: endothelin NOS (eNOS), inducible NOS (iNOS) and neuronal NOS (nNOS). Nitric oxide produced by eNOS plays an important role in the regulation of cell growth and programmed cell death as well as vasodilation and antithrombotic actions (31). Nitric oxide produced from eNOS from cardiomyocytes can enhance myocardial relaxation and regulate contractility as well as coronary perfusion $(32,33)$. These properties make eNOS regulation important in HF. In dogs with coronary microembolization-induced $\mathrm{HF}, \mathrm{mRNA}$ and protein expression of eNOS in LV myocardium is significantly downregulated compared to normal dogs (34). Long-term therapy with VNS significantly improves the expression of eNOS (34) (Fig. 5). 
Inducible NOS is expressed by many different cell types including inflammatory cells and cardiac myocytes. iNOS has been shown to be up-regulated in humans with HF (35). Ventricular unloading with LV assist device therapy has been shown to reduce the expression of iNOS and reduce cardiomyocyte apoptosis (35). Overexpression of iNOS in cardiomyocytes in mice has been shown to result in peroxynitrite generation associated with fibrosis, LV hypertrophy, chamber dilation and a cardiomyopathic phenotype (36). This overexpression also results in heart block and sudden cardiac death (36). In dogs with coronary microembolization-induced HF, mRNA and protein expression of iNOS in LV myocardium is significantly up-regulated compared to normal dogs (34). Long-term therapy with VNS tends to normalize the expression of iNOS in the failing dog LV myocardium (34) (Fig. 5).

Emerging evidence suggests that the neuronal form of NOS or nNOS is present in cardiomyocytes (37) and may be associated with the ryanodine receptor calcium release channel in the sarcoplasmic reticulum (38). This association suggests that nNOS may be capable of modulating calcium cycling and contractility (38). nNOS has been shown to be up-regulated in rats following myocardial infarctions as well as in human failing hearts (39). Studies in rats with HF have shown that preferential inhibition of nNOS leads to increased sensitivity of the myocardium to beta-adrenergic stimulation; a finding consistent with the concept that nNOS-derived NO production may play a role in the autocrine regulation of myocardial contractility (40). In dogs with coronary microembolization-induced HF, mRNA and protein expression of nNOS in LV myocardium was significantly up-regulated compared to normal dogs (34). Long-term therapy with VNS tends to normalize the expression of iNOS in the failing dog LV myocardium (34) (Fig. 5).

\section{Effects of VNS on Gap Junction Proteins in Dogs with Heart Failure}

Connexins, the protein molecules forming gap junctions, are reduced or redistributed from intercalated disks to lateral cell borders in a variety of cardiac diseases including HF (41). This "gap junction remodeling" is considered to be arrhythmogenic (41). Three principal connexins are expressed in cardiac myocytes, connexin-43 (Cx43), connexin-40 (Cx40) and connexin-45 (Cx45) (42). In adult mammalian ventricles, gap junctions exclusively contain Cx43 (43). Down-regulation of Cx43 (mRNA and protein) has been demonstrated in experimental HF models and in the failing human heart (44). Dephosphorylation of $\mathrm{Cx} 43$ has also been demonstrated in animal models of nonischemic HF (45). A heterogeneous $50 \%$ reduction in $\mathrm{Cx} 43$, similar to that of human heart failure, has been demonstrated to result in slowed transmural conduction and dispersion of action potential duration with increased susceptibility to arrhythmia (42) and sudden cardiac death. In dogs with coronary microembolization-induced HF, mRNA and protein expression of Cx43 in LV myocardium was markedly down-regulated compared to normal dogs (46). Long-term therapy with VNS was associated with a significant increase in the expression of Cx43 in LV myocardium of dogs with HF (46) (Fig. 6).

\section{Effects of VNS in Dogs with Rapid Pacing-Induced Heart Failure}

In contrast to the CardioFit system, the Cyberonics system used in dogs with HF secondary to high-rate ventricular pacing did not operate on a feedback mechanism. Instead, VNS therapy was delivered continuously for the duration of the study with a duty cycle of 14 seconds "on" and 12 seconds "off" (18). VNS signals were delivered at a frequency of $20 \mathrm{~Hz}$ and a pulse width of $0.5 \mathrm{~ms}$ (18). All dogs were implanted with a right ventricular pacemaker lead and were randomized to control $(n=7)$ or to VNS $(n=8)$. A right cervical Vagus stimulator was implanted in only the active VNS group. All dogs were followed for 8 weeks. In this study all data were collected with the animals in a sinus rate, approximately 
15 min after temporarily turning off the ventricular pacemaker and the Vagus nerve stimulator (18). In this canine model of HF, VNS therapy resulted in a significant decrease of LV end-diastolic and end-systolic volumes and a significant increase of LV ejection fraction compared to controls (18). The improvement in LV systolic function was associated with a significant reduction in plasma levels of norepinephrine, angiotensin-II and c-reactive protein. An important finding of this study was the demonstration that VNS can restore baroreflex sensitivity (18) thus improving cardiac autonomic control. Finally, the effects of VNS therapy in this model of HF can be viewed as being independent of heart rate because high-rate ventricular pacing was used to induce HF and the pacing rate was not affected by VNS (18).

\section{Conclusions and Clinical Implications}

A considerable body of pre-clinical investigations exists in support of the concept of electrical VNS as a therapeutic approach to treat of chronic HF. In animals with HF, VNS improves LV function and attenuates progressive LV remodeling and has the potential to suppress ventricular arrhythmias and prevent sudden cardiac death. VNS derives these potential clinical benefits from multiple mechanisms of action known to improve survival in patients with HF. These include reduced heart rate, attenuation of sympathetic over-drive and down-regulation of the renin-angiotensin-aldosterone system. In addition, VNS appears to have a positive impact on other signaling pathways that are likely to also elicit beneficial effects in patients with HF. These include restoration of baroreflex sensitivity, suppression of pro-inflammatory cytokines, normalization of nitric oxide signaling pathway and suppression of gap junction remodeling. At present, there is no evidence to implicate a single mechanism of action for the benefits derived from VNS. Instead, it is most likely that all of the mechanisms listed above act in concert to elicit the global benefit seen with VNS.

\section{Acknowledgments}

The authors wish to thank Drs. Ehud Cohen, Rami Biran, Tamir Ben-David and Yitzhak Sinai from BioControl Medical, Ltd. for their assistance on studies in dogs using the CardioFit VNS system.

Supported, in part, by research grants from BioControl Medical, Ltd. and National Heart, Lung, and Blood Institute PO1 HL074237-06

\section{References}

1. Schwartz PJ, Vanoli E, Stramba-Badiale M, De Ferrari GM, Billman GE, Foreman RD. Autonomic mechanisms and sudden death. New insights from the analysis of baroreceptor reflexes in conscious dogs with and without myocardial infarction. Circulation. 1988; 78:969-973. [PubMed: 3168199]

2. Mortara A, La Rovere MT, Pinna GD, Prpa A, Maestri R, Febo O, Pozzoli M, Opasich C, Tavazzi L. Arterial baroreflex modulation of heart rate in chronic heart failure: clinical and hemodynamic correlates and prognostic implications. Circulation. 1997; 96:3450-3458. [PubMed: 9396441]

3. La Rovere MT, Bigger JT, Marcus FI, Mortara A, Schwartz PJ. Baroreflex sensitivity and heart-rate variability in prediction of total cardiac mortality after myocardial infarction. Lancet. 1998; 351:478-484. [PubMed: 9482439]

4. Lechat P, Hulot JS, Escolano S, Mallet A, Leizorovicz A, Werhlen-Grandjean M, Pochmalicki G, Dargie H. on behalf of the CIBIS II Investigators. Heart rate and cardiac rhythm relationships with bisoprolol benefit in chronic heart failure in CIBIS II trial. Circulation. 2001; 103:1428-1433. [PubMed: 11245648]

5. Sabbah HN, Shimoyama H, Kono T, Gupta RS, Sharov VG, Scicli G, Levine TB, Goldstein S. Effects of long-term monotherapy with enalapril, metoprolol and digoxin on the progression of left ventricular dysfunction and dilation in dogs with reduced ejection fraction. Circulation. 1994; 89:2852-2859. [PubMed: 8205701] 
6. Sabbah HN, Stanley WC, Sharov VG, Mishima T, Tanimura M, Benedict CR, Hegde S, Goldstein $\mathrm{S}$. Effects of dopamine $\beta$-hydroxylase inhibition with nepicastat on the progression of left ventricular dysfunction and remodeling in dogs with chronic heart failure. Circulation. 2000; 102:1990-1995. [PubMed: 11034950]

7. Morita H, Suzuki G, Chaudhry PA, Anagnostopoulos PV, Tanhehco EJ, Sharov VG, Goldstein S, Sabbah HN. Effects of long-term monotherapy with metoprolol CR/XL on the progression of left ventricular dysfunction and remodeling in dogs with chronic heart failure. Cardiovasc Drugs and Therapy. 2002; 16:433-449.

8. Sabbah HN, Wang M, Jiang A, Ilsar I, Gupta RC, Rastogi S. Long-term Monotherapy with Ivabradine Improves Left Ventricular Function and Prevents Progressive Chamber Remodeling in Dogs with Moderate Heart Failure (Abstract). Circulation. 2009; 120:S867.

9. Kunze DL. Reflex discharge patterns of cardiac vagal efferent fibers. J Physiol (Lond). 1972; 222:1-8. [PubMed: 4338691]

10. Harman MA, Reeves TJ. Effects of efferent nerve stimulation on atrial and ventricular function. Am J Physiol. 1968; 215:1210-1217. [PubMed: 5687515]

11. Wiggers CJ. Physiology of the mammalian auricles: II. Influence of the vagus nerves on the fractionate contraction of the right auricle. Am J Physiol. 1916:133-140.

12. DeGeest H, Levy M, Zieske H, Lipman RI. Depression on ventricular contractility by stimulation of the vagus nerves. Circ Res. 1965; 17:222-235. [PubMed: 14338695]

13. Henry, JP.; Meehan, JP. Circulation: Integrative physiology study. Year Book Medical Publishers; Chicago: 1971.p. 57

14. Vanoli E, De Ferrari GM, Stramba-Badiale M, Hull SS Jr, Foreman RD, Schwartz PJ. Vagal stimulation and prevention of sudden death in conscious dogs with healed myocardial infarction. Circ Res. 1991; 68:1471-1481. [PubMed: 2019002]

15. Li M, Zheng C, Sato T, Kawada T, Sugimachi N, Sunagawa K. Vagal nerve stimulation markedly improves long-term survival after chronic heart failure in rats. Circulation. 2004; 109:120-124. [PubMed: 14662714]

16. Zheng C, Li M, Inagaki M, Kawada T, Sunagawa K, Sugimachi M. Vagal stimulation markedly suppresses arrhythmias in conscious rats with chronic heart failure after myocardial infarction. Conf. Proc IEEE Eng Med Biol Soc. 2005; 7:7072-7075. [PubMed: 17281904]

17. Sabbah HN, Stein PD, Kono T, Gheorghiade M, Levine TB, Jafri S, Hawkins ET, Goldstein S. A canine model of chronic heart failure produced by multiple sequential coronary microembolizations. Am J Physiol. 1991; 260:H1379-H1384. [PubMed: 1826414]

18. Zhang Y, Popovic ZB, Bibevski S, Fakhry I, Sica DA, Van Wagoner DR, Mazgalev TN. Chronic vagus nerve stimulation improves autonomic control and attenuates systemic inflammation and heart failure progression in a canine high-rate pacing model. Circulation. 2009; 2:692-699. [PubMed: 19919995]

19. Jones JFX, Wang Y, Jordan D. Activity of C fibre cardiac vagal efferents in anaesthetized cats and rats. Journal of Physiology. 1998; 507:869-880. [PubMed: 9508846]

20. Tosato M, Yoshida K, Toft E, Nekrasas V, Struijk. Closed-loop control of the heart rate by electrical stimulation of the vagus nerve. Med Biol Eng Comput. 2006; 44:161-169. [PubMed: 16937157]

21. Ben-Menachem E, Mañon-Espaillat R, Ristanovic R, Wilder BJ, Stefan H, Mirza W, Tarver WB, Wernicke JF. Vagus nerve stimulation or treatment of partial seizures: 1. A controlled study of effect on seizures. First International Vagus Nerve Stimulation Study Group. Epilepsia. 1994; 35:616-626. [PubMed: 8026408]

22. DeGiorgio C, Heck C, Bunch S, Britton J, Green P, Lancman M, Murphy J, Olejniczak P, Shih J, Arrambide S, Soss J. Vagus nerve stimulation for epilepsy: randomized comparison of three stimulation paradigms. Neurology. 2005; 65:317-319. [PubMed: 16043810]

23. De Herdt V, Boon P, Ceulemans B, Hauman H, Lagae L, Legros B, Sadzot B, Van Bogaert P, van Rijckevorsel K, Verhelst H, Vonck K. Vagus nerve stimulation for refractory epilepsy: a Belgian multicenter study. Eur J Paediatr Neurol. 2007; 11:261-269. [PubMed: 17395507]

24. Labiner DM, Ahern GL. Vagus nerve stimulation therapy in depression and epilepsy: therapeutic parameter settings. Acta Neurol Scand. 2007; 115:23-33. [PubMed: 17156262] 
25. Sabbah HN, Rastogi S, Mishra S, Gupta RC, Ilsar I, Imai M, Cohen U, Ben-David T, Ben-Ezra O. Long-term therapy with neuroselective electric Vagus nerve stimulation improves LV function and attenuates global LV remodelling in dogs with chronic heart failure. Europ J Heart Failure Supplements. 2005; 4:166. (Abstract).

26. Sabbah HN, Imai M, Zaretsky A, Rastogi S, Wang M, Jiang A, Zaca V. Therapy with Vagus nerve electrical stimulation combined with beta-blockade improves left ventricular function in dogs with heart failure beyond that seen with beta-blockade alone (Abstract). Europ J Heart Failure. 2007; 6:114.

27. Liu YH, Yang XP, Sharov VG, Nass O, Sabbah HN, Peterson E, Carretero OA. Effects of angiotensin-converting enzyme inhibitors and angiotensin ii type 1 receptor antagonists in rats with heart failure. Role of kinins and angiotensin ii type 2 receptors. J Clin Invest. 1997; 99:19261935. [PubMed: 9109437]

28. Tracey KJ. The inflammatory reflex. Nature. 2002; 420:853-859. [PubMed: 12490958]

29. Wang H, Yu M, Ochani M, Amella CA, Tanovic M, Susarla S, Li JH, Wang H, Yang H, Ulloa L, Czura CJ, Tracey KJ. Nicotinic acetylcholine receptor alpha7 subunit is an essential regulator of inflammation. Nature. 2003; 421:384-388. [PubMed: 12508119]

30. Gupta RC, Imai M, Jiang AJ, Wang M, Sabbah HN. Chronic therapy with selective electric vagus nerve stimulation normalizes plasma concentration of tissue necrosis factor-a, interleukin- 6 and Btype natriuretic peptide in dogs with heart failure (Abstract). J Am Coll Cardiol. 2006; 47:77A.

31. Feng Q, Song W, Lu X, Hamilton JA, Lei M, Peng T, Yee S-P. Development of Heart Failure and congenital septal defects in mice lacking endothelial nitric oxide synthase. Circulation. 2002; 106:873-879. [PubMed: 12176963]

32. Kelly RA, Balligand JL, Smith TW. Nitric oxide and cardiac function. Circ Res. 1996; 79:363380. [PubMed: 8781470]

33. Paulus WJ, Shah AM. NO and cardiac diastolic function. Cardiovasc Res. 1999; 43:595-606. [PubMed: 10690331]

34. Gupta RC, Mishra S, Rastogi S, Imai M, Zaca V, Sabbah HN. Chronic therapy with electric Vagus Nerve stimulation normalizes mRNA and protein expression of nitric oxide synthase in myocardium of dogs with heart failure (Abstract). Europ Heart J. 2006; 27:477.

35. Pattern RD, DeNofrio D, El-Zaru M, Kakkar R, Saunders J, Celestin F, Warner K, Rastegar H, Khabbaz KR, Udelson JE, Konstam MA, Karas RH. Ventricular assist device therapy normalizes inducible nitric oxide synthase expression and reduces cardiomyocyte apoptosis in the failing human heart. J Am Coll Cardiol. 2005; 45:1419-1424. [PubMed: 15862412]

36. Mungrue IN, Gros R, You X, Pirani A, Azad A, Csont T, Schulz R, Butany J, Stewart DJ, Husain M. Cardiomyocyte overexpression of iNOS in mice results in peroxynitrite generation, heart block and sudden death. J Clin Invest. 2002; 109:735-743. [PubMed: 11901182]

37. Xu KY, Huso DL, Dawson TM, Bredt DS, Becker LC. Nitric oxide synthase in cardiac sarcoplasmic reticulum. Proc Natl Acad Sci USA. 1999; 96:657-662. [PubMed: 9892689]

38. Barouch LA, Harrison RW, Skaf MW, Rosas GO, Cappola TP, Kobeissi ZA, Hobai IA, Lemmon CA, Burnett AL, O’Rourke B, Rodriguez ER, Huang PL, Lima JA, Berkowitz DE, Hare JM. Nitric oxide regulates the heart by special confinement of nitric oxide synthase isoforms. Nature. 2002; 416:337-339. [PubMed: 11907582]

39. Damy T, Ratajczak P, Shah AM, Camors E, Marty I, Hasenfuss G, Marotte F, Samuel JL, Heymes C. Increased neuronal nitric oxide synthase-derived NO production in the failing human heart. Lancet. 2004; 363:1365-1367. [PubMed: 15110495]

40. Bendall JK, Damy T, Ratajczak P, Loyer X, Monceau V, Marty I, Milliez P, Robidel E, Marotte F, Samuel J-L, Heymes C. Role of myocardial neuronal nitric oxide synthase-derived nitric oxide in $\beta$-adrenergic hyporesponsiveness after myocardial infarction-induced heart failure in rat. Circulation. 2004; 110:2368-2375. [PubMed: 15466641]

41. Jongsma HJ, Wilders R. Gap junctions in cardiovascular disease. Circ Res. 2000; 86:1193-1197. [PubMed: 10864907]

42. Severs NJ, Bruce AF, Dupont E, Rothery S. Remodelling of gap junctions and connexin expression in diseased myocardium. Cardiovasc Res. 2008; 80:9-19. [PubMed: 18519446] 
43. Van Kempen MJA, Ten Velde I, Wessels A, Oosthoek PW, Gros D, Jongsma HJ, Moorman AFM, Lamers WH. Differential connexins distribution accommodates cardiac function in different species. Microsc Res Tech. 1995; 31:420-436. [PubMed: 8534903]

44. Wang XJ, Gerdes AM. Chronic pressure overload cardiac hypertrophy and failure in guinea pigs: III. Intercalated disc remodeling. J Mol Cell Cardiol. 1999; 31:333-343. [PubMed: 10093046]

45. Ai X, Pogwizd M. Connexin 43 downregulation and dephosphorylation in nonischemic heart failure is associated with enhanced colocalized protein phosphatase type 2A. Circ Res. 2005; 96:54-63. [PubMed: 15576650]

46. Rastogi S, Mishra S, Ilsar I, Zaretsky A, Sabbah HN. Chronic therapy with electric Vagus nerve stimulation normalizes mRNA and protein expression of connexin-40, -43 and -45 in left ventricular myocardium of dogs with heart failure (Abstract). Circulation. 2007; 116:II-218. 


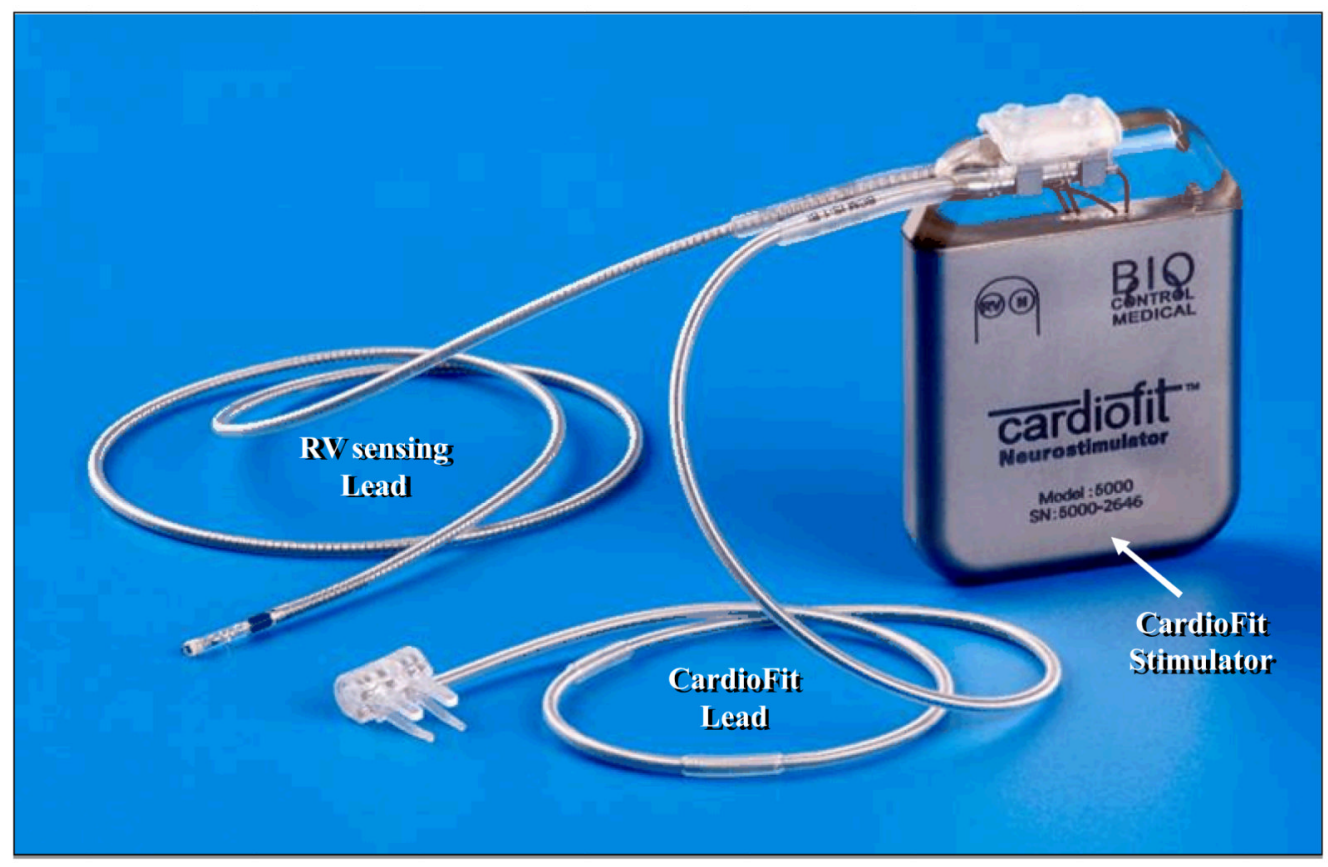

Figure 1.

Photograph of the BioControl Medical Vagus nerve stimulation device consisting of the signal generator (CardioFit stimulator), the right ventricular sensing lead, and the CardioFit right Vagus nerve cuff stimulation lead. 

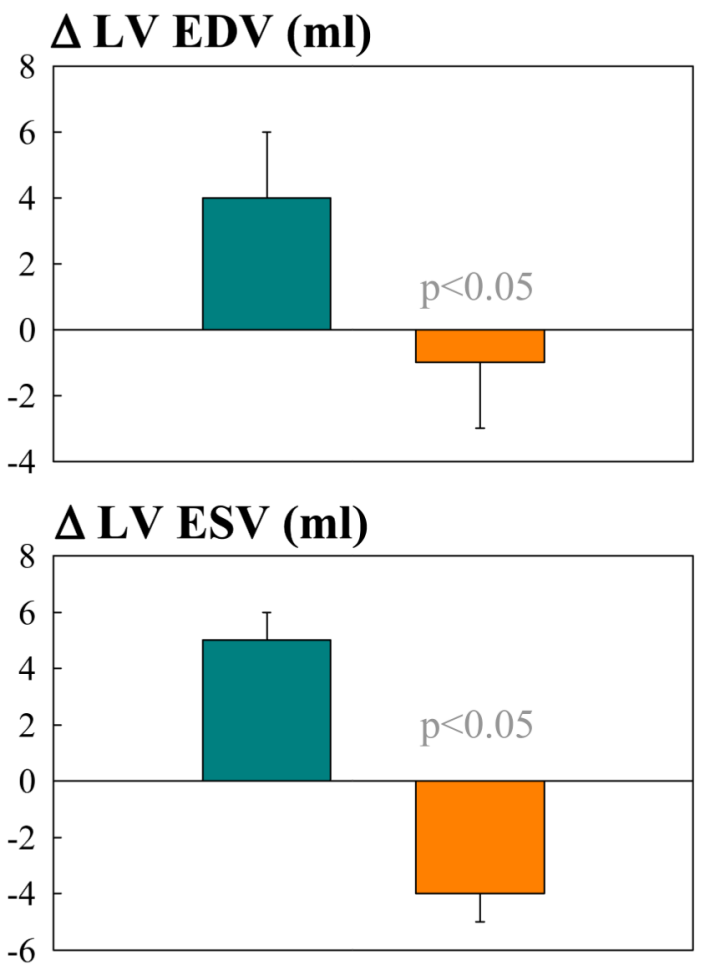

$\Delta$ LV EF (\%)

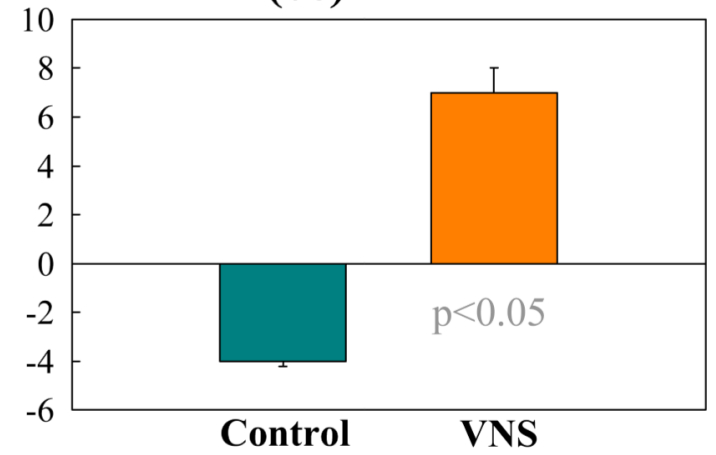

Figure 2.

Change $\Delta$ (treatment effect) between pre-treatment and post-treatment of left ventricular (LV) end-diastolic volume (EDV), end-systolic volume (ESV) and ejection fraction (EF) in untreated control dogs (blue bars) and dogs treated for 3 months with electric Vagus nerve stimulation (VNS). EDV and ESV measured from left ventriculograms using the area length method. 


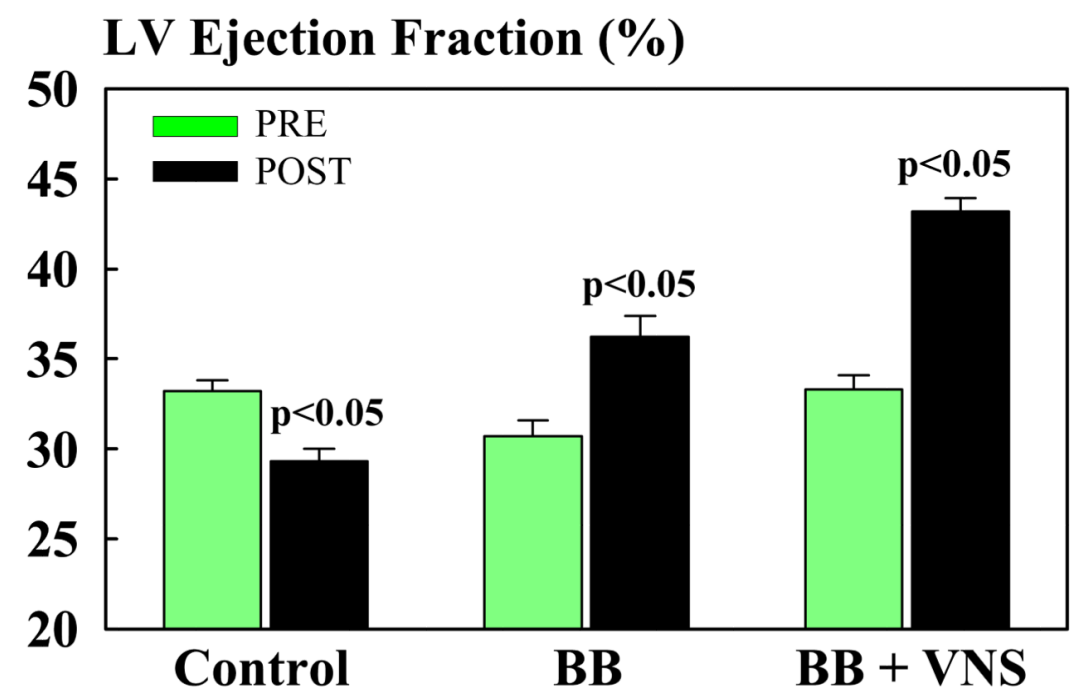

Figure 3.

Bar graph illustrating the change in left ventricular (LV) ejection fraction measured from ventriculograms before initiating therapy (PRE, green bars) and after 3 months of therapy (POST, black bars) in untreated heart failure control dogs, heart failure dogs treated with a beta blocker alone (BB, metoprolol succinate, $100 \mathrm{mg}$ once daily) and heart failure dogs treated with a combination of beta-blocker (metoprolol succinate, $100 \mathrm{mg}$ once daily) and electric Vagus nerve stimulation (VNS). 

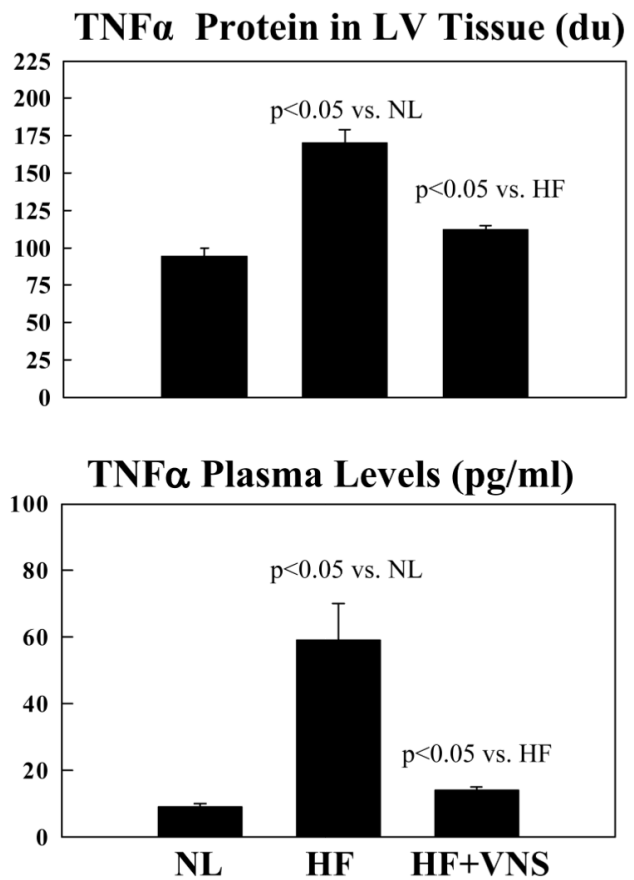

IL-6 Protein in LV Tissue (du)
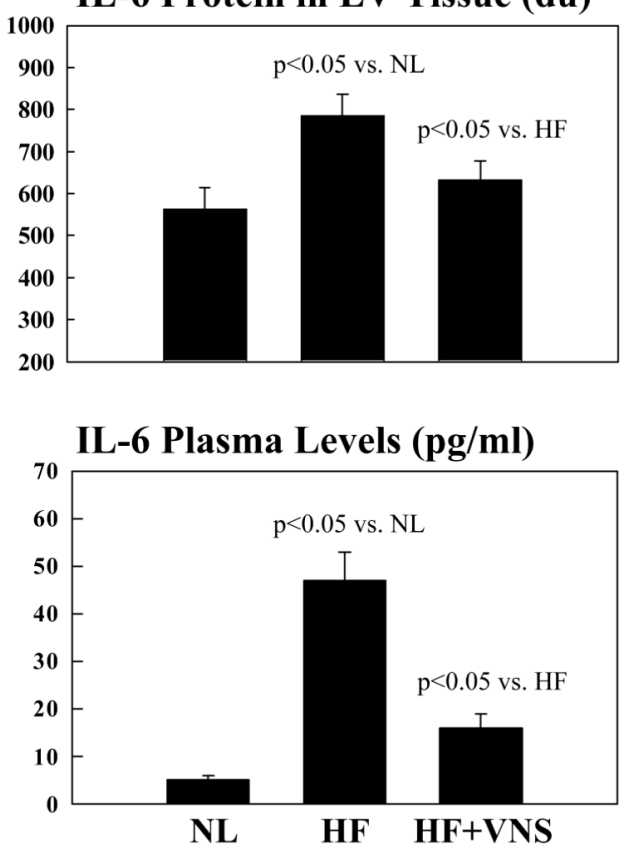

Figure 4.

TOP: Bar graphs illustrating the changes in tumor necrosis factor alpha (TNFa, top left) and interlukin-6 (IL-6, top right) in left ventricular myocardium of normal dogs (NL), untreated heart failure (HF) dogs and dogs with heart failure treated with electric Vagus nerve stimulation (HF+VNS). du = densitometric units.

BOTTOM: Bar graphs illustrating the changes in TNFa (bottom left) and IL-6 (bottom right) in plasma obtained from NL dogs, untreated HF dogs and dogs with HF treated with VNS (HF+VNS). 

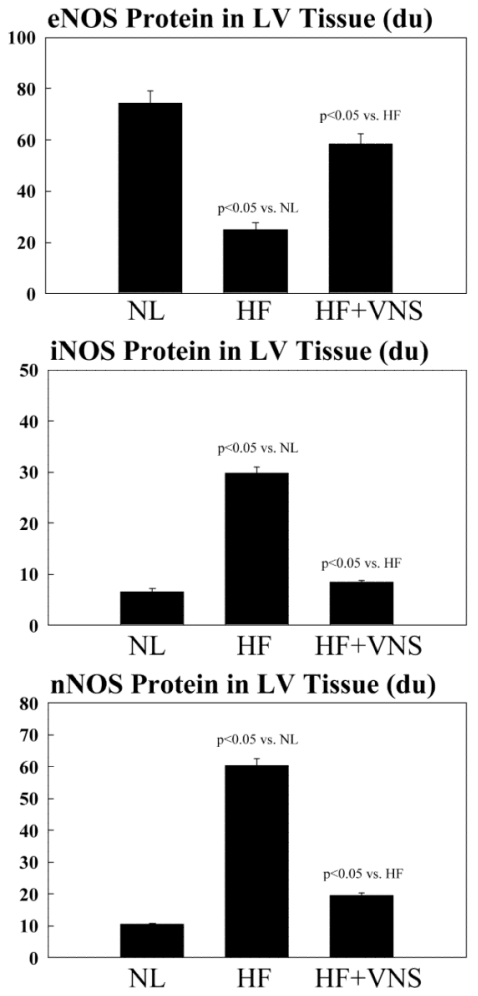

Figure 5.

TOP: Bar graph illustrating the changes in endothelial nitric oxide synthase (eNOS) in left ventricular myocardium of normal dogs (NL), untreated heart failure (HF) dogs and dogs with heart failure treated with electric Vagus nerve stimulation (HF+VNS). MIDDLE: Bar graph illustrating the changes in inducible nitric oxide synthase (iNOS). BOTTOM: Bar graph illustrating the changes in neuronal nitric oxide synthase (nNOS). du=densitometric units. 

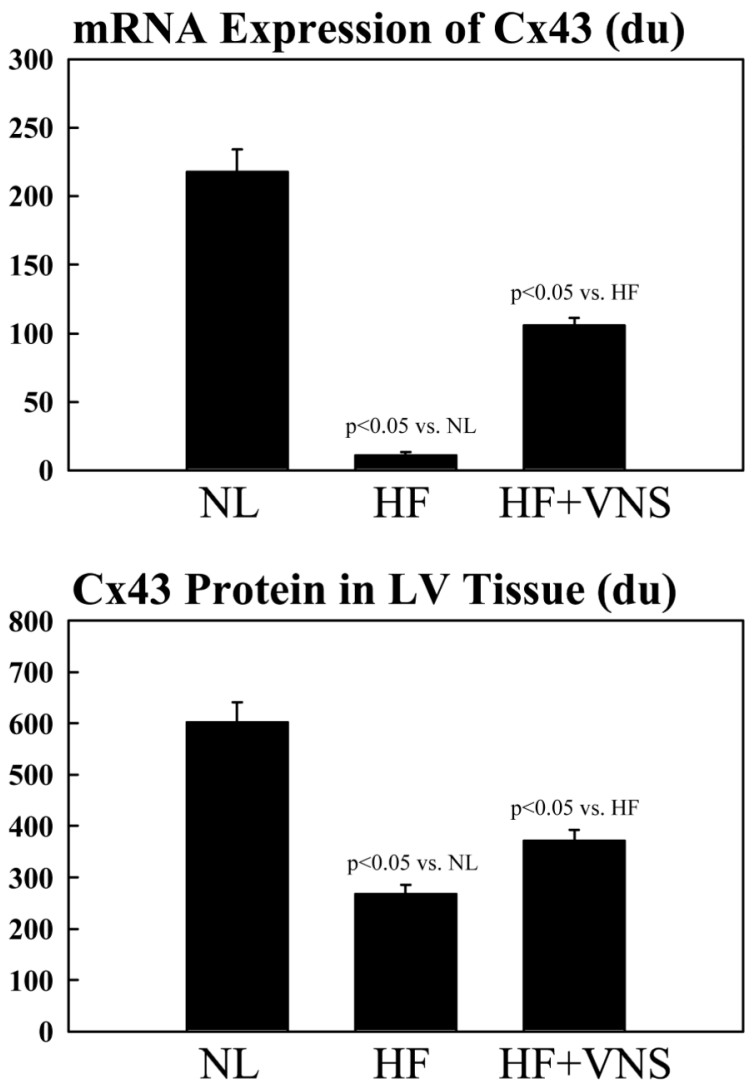

Figure 6.

TOP: Bar graph illustrating the changes in mRNA expression of connexin-43 (Cx43) in left ventricular myocardium of normal dogs (NL), untreated heart failure (HF) dogs and dogs with heart failure treated with electric Vagus nerve stimulation (HF+VNS). BOTTOM: Bar graph illustrating the changes in protein levels of $\mathrm{Cx} 43$ in left ventricular myocardium. $\mathrm{du}=$ densitometric units. 


\section{Table 1}

Indices of left ventricular diastolic function in control dogs and VNS-treated dogs obtained prior to initiating therapy (PRE) and at 3 months afterinitiating therapy (POST).

\begin{tabular}{|l|c|c|c|c|}
\hline \multirow{2}{*}{} & \multicolumn{2}{|c|}{ CONTROL $(\mathbf{n = 6})$} & \multicolumn{2}{c|}{ VNS $(\mathbf{n = 7})$} \\
\cline { 2 - 5 } & PRE & POST & PRE & POST \\
\hline LVEDP $(\mathrm{mmHg})$ & $14 \pm 1$ & $15 \pm 1$ & $15 \pm 1$ & $11 \pm 2^{*}$ \\
\hline PE/PA & $2.0 \pm 0.2$ & $1.8 \pm 0.2$ & $2.0 \pm 0.3$ & $2.4 \pm 0.4$ \\
\hline DT $(\mathrm{msec})$ & $91 \pm 1$ & $85 \pm 4$ & $94 \pm 5$ & $111 \pm 10^{*}$ \\
\hline EDWS $\left(\mathrm{gm} / \mathrm{cm}^{2}\right)$ & $54 \pm 3$ & $57 \pm 4$ & $60 \pm 5$ & $46 \pm 7^{*}$ \\
\hline
\end{tabular}

VNS=Vagus nerve stimulation; LVEDP=left ventricular end-diastolic pressure; $\mathrm{PE} / \mathrm{PA}=$ ratio of peak early rapid mitral inflow velocity (PE) to peak of mitral inflow velocity during left atrial contraction (PA); DT=deceleration time of early rapid mitral inflow velocity; EDWS=left ventricular end-diastolic circumferential wall stress.

* $=$ p $<0.05$ vs. PRE. 


\section{Table 2}

Plasma levels of n-terminal-pro brain natriuretic peptide (nt-pro BNP) in dogs with heart failure that were not treated (CONTROL) and dogs with heart failure treated with VNS.

\begin{tabular}{|l|c|c|c|}
\hline \multirow{2}{*}{} & \multicolumn{3}{|c|}{ nt-Pro BNP (fmols/ml) } \\
\cline { 2 - 4 } & Baseline & PRE & POST \\
\hline CONTROL & $212 \pm 18$ & $487 \pm 11^{*}$ & $546 \pm 126^{*}$ \\
\hline VNS & $230 \pm 18$ & $554 \pm 84^{*}$ & $260 \pm 24^{\dagger}$ \\
\hline
\end{tabular}

VNS=vagus nerve stimulation; Baseline=before initiating coronary microembolizations (normal); PRE=before initiating treatment; POST=3 months after initiating therapy or follow-up.

$=\mathrm{p}<0.05$ vs. Baseline;

${ }^{t}=\mathrm{p}<0.05$ vs. PRE. 


\section{Table 3}

Histomorphometric measures in left ventricular myocardium of normal dogs, dogs with heart failure that were not treated (CONTROL) and dogs with heart failure treated with VNS.

\begin{tabular}{|l|c|c|c|}
\hline & NORMAL $(\mathbf{n}=\mathbf{7})$ & CONTROL $(\mathbf{n}=\mathbf{6})$ & VNS $(\mathbf{n}=7)$ \\
\hline VFRF $(\%)$ & 0.0 & $21.0 \pm 2.0$ & $12.5 \pm 1.0^{*}$ \\
\hline VFIF $(\%)$ & $3.7 \pm 0.1$ & $8.3 \pm 0.2^{\dagger}$ & $6.5 \pm 0.3^{*}$ \\
\hline CD $\left(\right.$ Capillaries $\left./ \mathrm{mm}^{2}\right)$ & $2607 \pm 80$ & $1704 \pm 35^{\dagger}$ & $2403 \pm 73^{*}$ \\
\hline ODD $(\mu \mathrm{m})$ & $8.9 \pm 0.2$ & $11.0 \pm 0.1^{\dagger}$ & $9.2 \pm 0.1^{*}$ \\
\hline MCSA $\left(\mu \mathrm{m}^{2}\right)$ & $409 \pm 10$ & $720 \pm 14^{\dagger}$ & $549 \pm 15^{*}$ \\
\hline
\end{tabular}

VNS=Vagus nerve stimulation; $\mathrm{VFRF}=$ volume fraction of replacement fibrosis; VFIF=volume fraction of interstitial fibrosis; $\mathrm{CD}=$ capillary density; ODD=oxygendiffusion distance; MCSA=myocyte cross-sectional area.

${ }^{\dagger}=\mathrm{p}<0.05$ vs. NORMAL;

$=\mathrm{p}<0.05$ vs. CONTROL. 